Trinity University

Digital Commons @ Trinity

Psychology Faculty Research

Psychology Department

$12-2012$

\title{
Brooding Deficits in Memory: Focusing Attention Improves Subsequent Recall
}

\author{
Paula T. Hertel \\ Trinity University, phertel@trinity.edu \\ Amanda A. Benbow \\ Trinity University \\ E. Geraerts
}

Follow this and additional works at: https://digitalcommons.trinity.edu/psych_faculty

Part of the Psychology Commons

Publication Details

Cognition and Emotion

\section{Repository Citation}

Hertel, P.T., Benbow, A.A., \& Geraerts, E. (2012). Brooding deficits in memory: Focusing attention improves subsequent recall. Cognition and Emotion, 26(8), 1516-1525. doi: 10.1080/02699931.2012.668852

This Article is brought to you for free and open access by the Psychology Department at Digital Commons @ Trinity. It has been accepted for inclusion in Psychology Faculty Research by an authorized administrator of Digital Commons@ Trinity. For more information, please contact jcostanz@trinity.edu. 


\title{
BRIEF REPORT
}

\section{Brooding deficits in memory: Focusing attention improves subsequent recall}

\author{
Paula T. Hertel ${ }^{1}$, Amanda A. Benbow ${ }^{1}$, and Elke Geraerts ${ }^{2}$ \\ ${ }^{1}$ Department of Psychology, Trinity University, San Antonio, TX, USA \\ ${ }^{2}$ Department of Psychology, Erasmus University Rotterdam, Rotterdam, The Netherlands
}

\begin{abstract}
Ruminative habits of thought about one's problems and the resulting consequences are correlated with symptoms of depression and cognitive biases (Nolen-Hoeksema, Wisco, \& Lyubomirsky, 2008). In our orienting task, brooders and non-brooders concentrated on self-focusing phrases while they were also exposed to neutral target words. On each trial in the unfocused condition, participants saw and then reported the target before concentrating on the phrase; in the focused condition, the target was reported after phrase concentration. A brooding-related deficit on a subsequent unexpected test of free and forced recall was obtained in the unfocused condition only. Brooders recalled more successfully in the focused than in the unfocused condition. Thus, impaired recall of material unrelated to self-concerns may be corrected in situations that constrain attention.
\end{abstract}

Keywords: Memory; Recall; Rumination; Brooding.

Common experience suggests that people who become sidetracked by self-focused thoughts discover that there are negative memorial consequences for the neglected main event. We set out to test this hypothesis and to determine whether such a rumination-related memory deficit can be prevented by task support.

Rumination is the act of "thinking perseveratively about one's feelings and problems" (Nolen-Hoeksema, Wisco, \& Lyubomirsky, 2008, p. 400). Although rumination can exacerbate depressive symptoms and negative mood and predict the onset of major depressive episodes, it seems to be a stable trait over time, regardless of changes in depression levels. The Ruminative Responses Scale (RRS) of the Response Styles Questionnaire (Nolen-Hoeksema \& Morrow, 1991) is frequently used to assess self-reported tendencies to ruminate when sad. Treynor, Gonzalez, and Nolen-Hoeksema (2003) observed that many of the items on the RRS were similar in content to assessments of depressive symptoms. Therefore, in an effort to dissociate measures of rumination and depression, they identified

Correspondence should be addressed to: Paula T. Hertel, Department of Psychology, Trinity University, 1 Trinity Place, San Antonio, TX 78212, USA. E-mail: phertel@trinity.edu

The authors are grateful to Molly Holmes and Sarah Stec for their assistance in data collection.

(C) 2012 Psychology Press, an imprint of the Taylor \& Francis Group, an Informa business 
subscales of the RRS without obvious depressive content. One subscale-reflective ponderingseems to denote more adaptive ruminative tendencies, whereas a maladaptive form of rumination called brooding is more clearly associated with cognitive deficits.

Rumination or brooding is associated with cognitive costs, such as difficulty in setting aside negative thoughts (Joormann \& Gotlib, 2008) and disengaging attention from emotional material (Joormann, 2006). Ruminators have trouble forgetting emotional materials targeted for suppression practice (Hertel \& Gerstle, 2003) or occurring on a to-be-forgotten list (Joormann \& Tran, 2009). When brooders try to mentally refresh neutral words that they read moments earlier, they are slowed by the co-occurrence of emotional words competing for retrieval (Bernblum \& Mor, 2010). Rumination-inducing phrases have caused dysphoric students trouble in attention-demanding tasks such as randomnumber generation (Watkins \& Brown, 2002), Stroop colour naming (Philippot \& Brutoux, 2008), and controlled recollection of neutral materials from a prior task (Hertel, 1998). These are a few of the many reports documenting the cognitive costs of rumination or brooding when individuals are asked to concentrate on other matters. Although some of these reports concern memory phenomena, we are unaware of experimental demonstrations of memory deficits when the material to be remembered was encountered while participants were self focused, so we sought such evidence. The extent of concurrent processing of material irrelevant to selfconcerns should depend on tendencies to brood and thereby produce brooding-related differences on a test of subsequent recall. This prediction captures the proposed memorial consequences of becoming sidetracked from thinking about mundane events.

Our study also explored the possibility that such impairment is not a fixed feature of the cognitive abilities of brooders. We modelled the procedure on an experiment by Hertel and Rude (1991) in which clinically depressed, recovered, and control participants were presented with target words (e.g., artist) followed by sentence frames (e.g., The young man's physique was admired by the - ) and asked to judge the fit of the word in the sentence. The unfocused condition of this task, in which determination of fit could be made at any time, provided an opportunity for participants to ruminate during the eight seconds of phrase exposure on each trial. In the focused condition, however, the participants were asked to report both the target and the judgement of fit at the end of each eight-second interval. The advantage of focused attention was revealed on the unanticipated recall test, in that a depression-related deficit was found in the unfocused condition only. The unfocused condition was much like a typical orienting task involving decisions that encourage elaborative encoding. Showing a depression-related deficit in subsequent recall might be interpreted as providing evidence about reduced cognitive resources in depression (see Ellis \& Ashbrook, 1988) if it were not for the fact that depressed individuals were capable of performing as well as non-depressed when the orienting task constrained attention to the task at hand. Instead of reduced resources, attentional control appeared to be the critical factor. And one possibility is that rumination was the culprit that was overpowered by such control.

A cursory reading of the literature on rumination and cognition might likewise give the impression that ruminative thoughts, prevalent in depression, impair cognitive resources. To address that possibility, we attempted to overcome possible resource deficits through the experimental control of attention. To encourage self-focus, we exposed participants to rumination-inducing phrases (Nolen-Hoeksema \& Morrow, 1993) during the periods between target presentations. We hoped to show that resulting brooding-related memory deficits are not inevitable, as a resource-depletion account might suggest, because they can be prevented by constraining attention during the orienting task (much liked the focused condition used by Hertel \& Rude, 1991). In that regard, we predicted an interaction between brooding group and the type

COGNITION AND EMOTION, 2012, 26 (8) 
of orienting task, expecting a larger broodingrelated deficit in recall in the unfocused condition than in the focused condition.

Secondarily and on an exploratory basis, we examined the phenomenological consequences of experimental control of attention. Other attempts to change affective states as a consequence of rumination interventions have been successful (see Nolen-Hoeksema et al., 2008; Watkins, 2008). Therefore it seemed possible that experience in learning to mentally maintain self-irrelevant material might improve mood and increase feelings of competence and self-control. To explore this possibility, we collected ratings before and after the orienting task.

\section{METHOD}

\section{Participants}

Ninety-six undergraduate students at Trinity University participated in the experiment for extra course credit. Approximately 1-3 weeks prior to their participation, students completed the RRS in their introductory class and, unbeknownst to them, were selected to participate according to their brooding score (Treynor et al., 2003). Median brooding scores varied between 9 and 10, depending on the class surveyed. Participants with brooding scores above 10 were selected as brooders and those with scores below 9 as nonbrooders. Under the constraint of equal cell size, participants within each group were randomly assigned to either the focused or unfocused condition. Group membership was ultimately determined by the same criteria applied to the score at the end of the session; the data from the 24 participants who scored 9 or 10 on the second administration were omitted from the main analyses but included in the correlational analysis reported subsequently. The female-to-male ratio was 13:5 in each condition, except brooders in the focused condition, where the ratio was 14:4.

\section{Materials}

Phrases and target words. We selected 30 phrases from Nolen-Hoeksema and Morrow's (1993) rumination-induction procedure. All phrases were designed to focus attention on oneself (e.g., How you feel about your friendships; Why you react the way you do; The possible consequences of your current mental state). To facilitate recall, we selected 10 categories of three words each from Van Overshelde, Rawson, and Dunlosky's (2004) category norms (e.g., flower: pansy, orchid, petunia; type of bird: pigeon, seagull, canary). Individual words were selected in order to balance the categories according to the proportion of participants who provided the word when cued with the category in the norming study $(M=0.12)$, word frequency $(M=26)$, and word length $(M=5.67){ }^{1}$

Visual Analogue Scales (VAS). A set of unidimensional VAS was used to assess self-reported competence, control, and happiness. Participants indicated their "current state of mind" by drawing a vertical line through a horizontal line $100 \mathrm{~mm}$ in length that represented each dimension. The dimensions were anchored by incompetent and very competent, out of control and very much in control, sad and happy.

Assessment methods. The Beck Depression Inventory (BDI-II) is a 21-item self-report measure used to assess depressive symptoms (Beck, Steer, \& Brown, 1996). We omitted the item related to suicide (as requested by our institutional review board); possible scores therefore ranged from 0 to 60. The RRS is a 22-item measure designed by Nolen-Hoeksema and Morrow (1991) to assess trait rumination. We defined participant groups based on scores from the 5 -item brooding subscale identified by Treynor et al. (2003). Scores on both the brooding and reflective-pondering subscales could range from 5 to 20; possible RRS scores

\footnotetext{
${ }^{1}$ Category clustering in recall did not differ according to condition or group.
}

\section{COGNITION AND EMOTION, 2012, $26(8)$}


range from 22 to 88 . Table 1 presents means and standard deviations.

\section{Orienting task}

The orienting task (programmed in Superlab Pro 4.07, Cedrus Corporation, San Pedro, CA) consisted of two sections of 30 trials each. The 30 target words and 30 phrases were randomly paired for presentation within each section, with no duplication of pairing across sections. (All materials were presented twice because we were concerned about floor effects in subsequent recall.) Each block of 10 trials contained one word from each of the 10 categories, with trials randomised within blocks. The first block began with two practice trials to ensure comprehension of instructions.

On each trial in the unfocused condition, participants first saw a target word for $1,500 \mathrm{~ms}$, followed by a plus sign displayed in the centre of the screen for $500 \mathrm{~ms}$. Then "word?" signalled them to type the target in a box at the bottom of the screen and press the enter key. If the participant failed to press enter within $5 \mathrm{~s}$ of the request, the program automatically moved on. To ensure conceptual processing of the target, the next screen requested a rating of its pleasantness $(1=$ Unpleasant to $7=$ Pleasant $)$. A phrase was then displayed for $11 \mathrm{~s}$, turning blue in the last $3 \mathrm{~s}$ in order to signal the upcoming rating of the degree of tension or relaxation the participant felt in response to concentrating on the phrase $(1=$ Tense to $7=$ Relaxed $)$. The purpose of this rating was the provision of a rationale for attending to the phrases. After the first block of 30 trials, an instruction screen explained that the next set of 30 trials would include a second rating for each idea and a concomitantly longer exposure time (which was $15 \mathrm{~s}$ ). After rating relaxation, the participants rated ease of concentrating on the phrase $(1=$ Difficult to $7=$ Easy).

The trial events were the same in the focused condition, but their order differed. The target was followed by the phrase and the rating of tension or relaxation (and concentration in the second section). Then "word?" was displayed to cue typing of the target, which was followed by the rating of its pleasantness.

\section{General procedure}

Participants first responded to the VAS form. Next, in the orienting task, participants were

Table 1. Mean scores on the self-report measures (standard deviations)

\begin{tabular}{lccrc}
\hline Measure & Unfocused non-brooders & Unfocused brooders & Focused non-brooders & Focused brooders \\
\hline BDI-II & $5.5(4.44)$ & $16.6(9.31)$ & $6.4(4.62)$ & $15.6(8.54)$ \\
RRS & $31.1(6.24)$ & $56.8(7.96)$ & $32.6(4.54)$ & $53.2(9.46)$ \\
$\quad$ Brooding subscale & $6.5(0.92)$ & $13.8(2.16)$ & $6.9(0.90)$ & $13.7(2.09)$ \\
$\quad$ Pondering subscale & $7.7(2.65)$ & $12.2(2.53)$ & $7.4(1.68)$ & $11.1(2.99)$ \\
VAS competence & & & & $75.0(16.3)$ \\
$\quad$ Pre-task & $80.3(15.3)$ & $67.9(18.8)$ & $59.2(27.0)$ & $74.2(18.4)$ \\
$\quad$ Post-task & $80.3(14.8)$ & $56.6(18.5)$ & & $62.0(22.1)$ \\
VAS control & & & $77.4(18.1)$ & $74.8(21.2)$ \\
$\quad$ Pre-task & $85.8(15.3)$ & $66.5(21.4)$ & $62.2(24.0)$ & $61.6(23.3)$ \\
$\quad$ Post-task & $81.4(17.1)$ & $52.0(16.3)$ & & $79.9(18.6)$ \\
$\quad$ VAS happiness & & & $72.7(16.1)$ & $69.4(19.0)$ \\
$\quad$ Pre-task & $82.8(12.0)$ & $61.1(21.9)$ & $45.7(21.8)$ & $5.9(25.8)$ \\
\hline
\end{tabular}

Notes: $n=18$ for the BDI and RRS scores; $n=17$ for the VAS measures. All questionnaire scores differed significantly only according to group. BDI-II: $F(1,68)=36.91, M S E=50.14, p<.001, \eta_{\mathrm{p}}^{2}=.35 ;$ RRS: $F(1,68)=182.3, \quad M S E=53.13, p<.001, \eta_{\mathrm{p}}^{2}=.73$; Pondering: $F(1,68)=47.66, M S E=6.30, p<.001, \eta_{\mathrm{p}}^{2}=.41$. (Brooding scores were experimentally constrained.) All other effects for questionnaire scores, $p>.244$. 
instructed to read each phrase slowly and to use their imagination and concentration to focus on each one for the entire time that it was displayed. When the phrase switched to a blue colour, this was the signal that it was almost time to rate how tense or relaxed the idea made them feel. They were also told that "divider words" (our target words) would appear briefly between the ideas. Completely unrelated to the ideas, divider words and the corresponding rating of pleasantness should help them take their mind off the previous idea and prepare them to focus on the next one. Further instructions about the rating of ease of concentration were delivered between the two sections of the task.

After completing the orienting task, participants again responded to the VAS form. They were then asked to recall as many target words as possible. When they indicated that they were finished recalling, they were instructed to draw a line under the last word on their sheet, continue recalling until a set number of words was reached (either 20 or 25$),{ }^{2}$ and guess if necessary. This procedure was used to counteract possible response bias. Next, participants took a readingspan test ${ }^{3}$ before responding to the BDI-II and the RRS. All VAS and assessment forms were collected by the participant in an envelope not to be viewed by the experimenter.

\section{RESULTS}

Data collected on the following measures were submitted to analyses of variance (ANOVAs): number of target words freely recalled, total number recalled (free and forced), number of errors made in reporting the targets during the orienting task, rated ease of concentration on the phrases, and VAS ratings. Each ANOVA included between-subjects factors for Group (non-brooders vs. brooders) and Task Condition (unfocused vs. focused). To more clearly define the groups, data for these analyses excluded participants whose end-of-session brooding scores were 9 or 10 (medians on class administrations). For all analyses, the significance level was set at .05. Statistics for significant main effects that were qualified by significant interactions are not reported. In analyses that also include withinsubjects factors (number of errors and VAS ratings), Huynh-Feldt adjustments were used under violations of sphericity. Finally, correlations between recall measures and scores on the BDI and RRS were performed on data from all 96 participants.

\section{Test phase}

Free recall. As is illustrated in Figure 1, the analysis of the number of target words freely recalled revealed a significant interaction, $F(1$, 68) $=5.92, M S E=16.56, p=.018, \eta_{\mathrm{p}}^{2}=.08$. In the unfocused condition, brooders recalled significantly fewer targets than non-brooders,

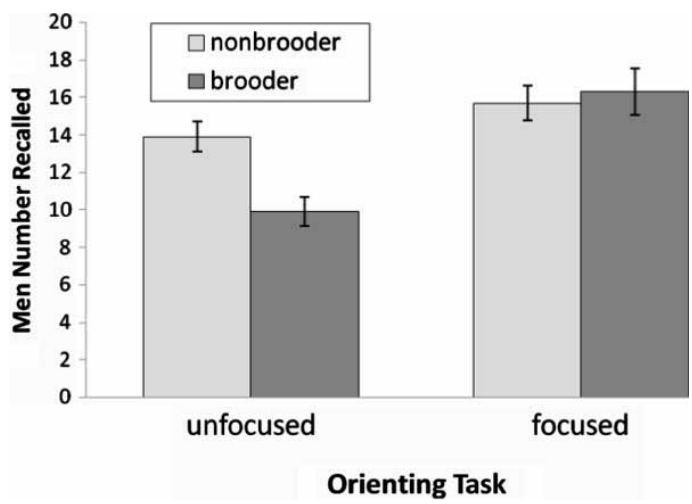

Figure 1. Mean number of words freely recalled by non-brooders and brooders in the focused and unfocused conditions of the orienting task. Error bars represent one standard error.

\footnotetext{
${ }^{2}$ The first 29 participants, evenly distributed across conditions, were instructed to recall at least 20 words. Subsequently, we raised the criterion to 25 words. An analysis that included criterion as a factor in the full design failed to reveal significant interaction effects involving criterion, $F<1.0$ for all three. Free recall was non-significantly higher with the lower criterion, $p=.068$.

${ }^{3}$ This task was incorporated as an exploratory measure of transfer; reading-span scores were not significantly related to the main variables in this report.
} 
$F(1,68)=8.94, p=.004, \eta_{\mathrm{p}}^{2}=.12$, whereas in the focused condition, the two groups recalled similarly, $F<1.0$. Viewed from the alternative perspective, brooders clearly profited from the focusing operation, $F(1,68)=22.18, p<.001$, $\eta_{\mathrm{p}}^{2}=.25$, whereas non-brooders did not, $F(1,68)=1.61, p=.209$.

Total recall. The analysis of the total number of words recalled - before and after participants were required to write to a criterion-produced similar outcomes to those reported for free recall. The interaction was significant, $F(1,68)=4.82$, $M S E=18.89, p=.031, \eta_{\mathrm{p}}^{2}=.07$. Tests of simple main effects revealed a brooding deficit within the unfocused condition, $F(1,68)=8.95, p=.004$, $\eta_{\mathrm{p}}^{2}=.12 \quad\left(M_{\text {non-brooders }}=18.4, \quad S D=4.29\right.$; $\left.M_{\text {brooders }}=14.1, S D=4.07\right)$, but not the focused condition, $\quad F<1.0 \quad\left(M_{\text {non-brooders }}=19 . \quad 8\right.$, $\left.S D=4.43 ; \quad M_{\text {brooders }}=19.9, \quad S D=4.58\right)$. From the other perspective, brooders were helped by the focusing operation, $F(1,68)=16.22$, $p<.001, \eta_{\mathrm{p}}^{2}=.19$, but non-brooders were not, $F<1.0$. These outcomes suggest that the results for free recall did not reflect a significant degree of response bias.

\section{Orienting task}

Reporting errors. As a measure of ability to comply with task demands, we analysed the percentage of errors in reporting the target words within each of the six blocks of 10 consecutive trials. The analysis revealed a main effect of Block, $F(3,60,245.98)=3.66, M S E=81.80, p=.009$, $\eta_{\mathrm{p}}^{2}=.05$, and a corresponding significant linear trend across blocks, $F(1,68)=5.83$, $M S E=114.06, p=.018, \eta_{\mathrm{p}}^{2}=.08$. The percentage of reporting errors increased over time, reflecting either fatigue or increased difficulty in the second half due to the longer phrase durations. As expected, those in the focused condition made more errors overall than those in the unfocused condition, $F(1, \quad 68)=42.91, \quad M S E=323.84$, $p<.001, \eta_{\mathrm{p}}^{2}=.39\left(M_{\text {unfocused }}=1,2, S D=2.92\right.$; $\left.M_{\text {focused }}=12.5, \quad S D=9.88\right) .{ }^{4}$ All other effects were non-significant, $p>.353$. Thus, brooders and non-brooders were equally able to report the targets.

Ease of concentration. Following the relaxation rating on trials during the second half of the orienting task, participants reported how easy it was for them to concentrate on the phrase. Lower ratings reflected greater difficulty. The analysis revealed a significant main effect of Group, $F(1$, $68)=6.46, \quad M S E=2.03, \quad p=.013, \quad \eta_{\mathrm{p}}^{2}=.09$. Non-brooders reported greater ease in concentrating on the phrases $\left(M_{\text {non-brooder }}=4.9, S D=0.77\right.$; $\left.M_{\text {brooder }}=4.4, \quad S D=0.88\right)$, suggesting greater comfort with self-focused thoughts. No other effects were significant, $p>.084$. In particular, the lack of a Group by Task interaction $(p=.995)$ suggests that brooders' perceived difficulties were not differentially greater in the focused condition as a consequence of the requirement to report targets subsequently.

\section{Visual Analogue Scales}

VAS ratings were submitted to a mixed-design ANOVA, with within-subjects factors for Time (pre and post orienting task) and Dimension (competence, control, and happiness) and between-subjects factors for Group and Task. Means are reported in Table 1 . The only significant effect involving Dimension in the overall analysis was its interaction with Group, $F(1,74$, $116.30)=6.05, \quad M S E=223.30, \quad p=.005$, $\eta_{\mathrm{p}}^{2}=.08$. Across time and task conditions, the brooding-related deficit was greatest for happiness (20.0 pts on average), next greatest for feelings of control (13.0 pts), and least for competence (8.5 pts), but each of the three dimensions revealed significant brooding deficits, $p<.040$.

Across administration times and dimensions, group differences in ratings depended on the Task Condition, $F(1,67)=6.94, \quad M S E=1,454.73$,

\footnotetext{
${ }^{4}$ Spelling errors were not included as errors. Errors included omissions, other targets, and confabulations, even in the unfocused condition.
} 
$p=.010, \eta_{\mathrm{p}}^{2}=.09$. Moreover, the three-way interaction of Group, Condition, and Time showed a non-significant trend, $F(1,67)=3.47$, $M S E=297.12, p=.067, \eta_{\mathrm{p}}^{2}=.05$. In the unfocused condition, the interaction of Time and Group, $\quad F(1, \quad 34)=5.88, \quad M S E=299.92$, $p=.021, \quad \eta_{\mathrm{p}}^{2}=.15$, reflected brooders' greater drop along all dimensions after the orienting task. Other interaction effects in the unfocused condition were non-significant, $p>.168$. In contrast, non-brooders and brooders felt similarly and less positively following the focused condition of the orienting task, $F(1,33)=26.43$, $M S E=294.23, \quad p<.001, \eta_{\mathrm{p}}^{2}=.44$. All effects within the focused condition were non-significant, with the exception of a Group by Dimension interaction indicating that brooders were generally less happy but not feeling less competent or in control than non-brooders, $F(2,66)=3.87$, $M S E=199.90, p=.026, \eta_{\mathrm{p}}^{2}=.10$.

\section{Correlations}

The previously reported analyses were based on data from students whose brooding scores met the classification criteria; however, data were also collected from 24 students whose end-of-session scores were 9 and 10. By including these data we examined correlations within each task condition between measures of recall and the scores on the BDI and RRS. ${ }^{5}$ As reported in Table 2, we found that brooding and RRS scores were significantly correlated with the number of targets freely recalled in the unfocused condition, but not in the focused condition. The same pattern of significance was obtained with total recall. We also call attention to the non-significant correlations between recall measures and BDI-II scores in the unfocused condition, despite the fact that BDI-II scores were positively correlated with total RRS scores and scores from the reflectivepondering and brooding subscales, as is typically found; with RRS, $r(94)=.69, p<.001$; with brooding, $r(94)=.59, p<.001$, with pondering, $r(94)=.43, p<.001$. Thus, dysphoria was not a mediating variable in our results.

\section{DISCUSSION}

In asking our participants to concentrate on selffocused phrases, we expected to divert attention from target words by those with tendencies to brood. Indeed, the participants in the brooding group were sufficiently inattentive to the targets that later they recalled them poorly. This brooding-related difference was not inevitable, however, because the simple requirement to report each target a few seconds following its presentation was sufficient to prevent the recall deficit. These effects were obtained both by group analyses that omitted data from borderline brooders and by correlational analyses that included all data. The correlational approach also allowed us to rule out dysphoria as a factor responsible for the relation between brooding and recall. These

Table 2. Correlations between free recall and measures of dysphoria (BDI-II) and rumination (RRS)

\begin{tabular}{lcc}
\hline Measure & Unfocused task & Focused task \\
\hline BDI-II & -.26 & -.08 \\
RRS & $-.39^{*}$ & .06 \\
$\quad$ Brooding & $-.42^{*}$ & .06 \\
$\quad$ Pondering & -.26 & .09 \\
\hline
\end{tabular}

Notes: ${ }^{*} p<.01 . n=45$ in the unfocused condition and 51 in the focused condition.

\footnotetext{
${ }^{5}$ Interpretation of these correlations should consider the fact that individuals who scored 9 and 10 during screening were not included in the sample. Regression analyses were also performed on the number of targets freely recalled by allowing the main effects (a code for condition and the brooding score) to enter first, followed by their interaction. The first equation contained only the main effect of condition, $R=.375, F(1,94)=15.40, p<.001$; the full equation also included the interaction parameter, $R=.427, F(2,93)=10.40, p<.001$. The change in $R^{2}$ with the interaction was significant, $F(1,93)=4.77, p=.031$. Two separate and similar analyses were also performed with BDI-II scores and reflective pondering scores contributing to interaction predictors. Neither interaction predictor produced a significant change in $R^{2}$ beyond the condition code $(p=.447$ for BDI-II and $p=.106$ for reflective pondering).
} 
results therefore fit within a small body of evidence for associations between measures of performance on a cognitive task (typically attention tasks) and rumination, in the absence of similar associations with measures of depression (e.g., Daches, Mor, Winquist, \& GilboaSchechtman, 2010; de Lissnyder, Derakshan, de Raedt, \& Koster, 2011).

Our interpretation of the recall data is based on the idea that the "divider words" (targets) engender less conceptual processing from brooders in the unfocused condition, while they are concentrating on the self-focused phrases. Clearly, this result can be viewed as a deficiency in processing the targets or as effective filtering of less important material, given the nature of our instructions to the participants. (Divider words are used to clear one's mind.) These alternatives align with differences in common experience. The incidental features of a routine task to be performed might seem much less important than the topic of one's rumination. On the other hand, individuals who don't typically focus on themselves might welcome the opportunity to think about mundane matters. These possibilities are at the centre of what it means to experience a task that does not constrain the focus of attention (see Hertel \& Rude, 1991). The tendency to attend is much more the issue than the ability to attend, because when the task constrains attention to potentially minor features like our divider words, brooders remember them well.

A possible limitation on our conclusions is the absence of a measure of rumination. Because it was unclear how such a measure could be obtained during or after the orienting task without interfering with recall, we relied on the bootstrapping procedure of employing materials that are assumed to function as inductions of state rumination (see Nolen-Hoeksema et al., 2008). Another possible limitation on our conclusions concerns the lack of variation in the nature of the phrases we used. We would like to say that selffocus engendered by the rumination-inducing phrases (Nolen-Hoeksema \& Morrow, 1993) is responsible for the brooding-related deficit, but without conditions employing other types of material for concentration, we cannot be confident that the self-focused phrases were necessary for the effect. Although the results in the focused condition suggest that brooders do not experience fundamental attentional difficulties, without task support it is possible that brooders would recall targets poorly, regardless of the nature of the material for concentration. This issue should be addressed experimentally.

Our emphasis on rumination as a primary predictor is partly based on previous evidence of a causal role for state rumination in the relation between dysphoria and controlled recollection (Hertel, 1998): Dysphoric students who were assigned to contemplate ruminative phrases between the orienting task and the test showed the same deficit as those who merely waited seven minutes for the test (and a distraction condition eliminated it). Those results implicated impaired retrieval processes, whereas the current results point to processes operating during the orienting task. Our results are also related to those described by Bernblum and Mor (2010), whose use of a "refresh" task revealed that brooders experienced interference from negative words presented along with the neutral words they were attempting to recognise moments later. We note that like their negative words our self-focused phrases might have made it difficult for brooders to hold the neutral target words in mind or to bring them immediately back to mind (refresh them) during the focused orienting task; our measure of reporting errors might have been insufficiently sensitive to such possibilities.

The accuracy of target reports during the orienting task did not differ according to brooding tendencies. It is possible that brooders in the focused condition differentially sacrificed attention to the phrases in order to report the targets and, compared to non-brooders, brooders did experience greater difficulty in phrase concentration (although not more so in the focused condition). Reporting errors and concentration ratings, however, are relatively insensitive measures of attention to the words and phrases. Therefore, we conclude that brooders appear to be capable of staying on task when the task is

COGNITION AND EMOTION, 2012, 26 (8)

1523 
structured to encourage it, but recommend the use of more sensitive measures in the future. Alternatively, it is possible that recruiting more extreme groups of brooders would reveal differences in measures taken during and following the focused task.

Finally, being required to stay on task did not significantly incur feelings of competence and control, as we initially thought it might. As expected, brooders were more negatively affected than non-brooders by the unfocused orienting task (pre vs. post task). But even though the effect of the focused task was similar for the two groups, it was not significantly reduced compared to the effect in the unfocused condition. The increasing number of errors in reporting the targets across blocks might be at least partly responsible for lack of improvement in mental well-being. In spite of our lack of success, we recommend that such improvement be sought in the future. Other types of research strategies for counteracting rumination seem to improve well-being. For example, Watkins, Baeyens, and Read (2009) found that concreteness training - a form of cognitive-bias modification geared toward changing the nature of ruminative tendencies - decreased depressive symptoms in a dysphoric group. Efforts to improve experimental support for attentional control might lead to successful training of perceived self-control and competence. These efforts are worth making, because feelings of competence and control should reduce the tendency to ruminate in the first place.

Manuscript received 6 September 2011 Revised manuscript received 12 January 2012

Manuscript accepted 17 January 2012

First published online 7 June 2012

\section{REFERENCES}

Beck, A. T., Steer, R. A., \& Brown, G. K. (1996). Manual for the Beck Depression Inventory (2nd ed.). San Antonio, TX: Psychological Corporation.
Bernblum, R., \& Mor, N. (2010). Rumination and emotion-related biases in refreshing information. Emotion, 10, 423-432.

Daches, S., Mor, N., Winquist, J., \& GilboaSchechtman, E. (2010). Brooding and attentional control in processing self-encoded information: Evidence from a modified Garner task. Cognition and Emotion, 24, 876-885.

de Lissnyder, E., Derakshan, N., de Raedt, R., \& Koster, E. (2011). Depressive symptoms and cognitive control in a mixed antisaccade task: Specific effects of depressive rumination. Cognition and Emotion, 25, 886-897.

Ellis, H. C., \& Ashbrook, P. W. (1988). Resource allocation model of the effects of depressed mood states on memory. In K. Fiedler \& J. Forgas (Eds.), Affect, cognition and social behavior (pp. 25-43). Toronto, Canada: Hogrefe.

Hertel, P. T. (1998). Relation between rumination and impaired memory in dysphoric moods. Journal of Abnormal Psychology, 107, 166-172.

Hertel, P. T., \& Gerstle, M. (2003). Depressive deficits in forgetting. Psychological Science, 14, 573-578.

Hertel, P. T., \& Rude, S. S. (1991). Depressive deficits in memory: Focusing attention improves subsequent recall. Journal of Experimental Psychology: General, 120, 301-309.

Joormann, J. (2006). Differential effects of rumination and dysphoria on the inhibition of irrelevant emotional material: Evidence from a negative priming task. Cognitive Therapy \& Research, 30, 149-160.

Joormann, J., \& Gotlib, I. H. (2008). Updating the contents of working memory in depression: Interference from irrelevant negative material. Journal of Abnormal Psychology, 117, 182-192.

Joormann, J., \& Tran, T. B. (2009). Rumination and intentional forgetting of emotional material. Cognition and Emotion, 23, 1233-1246.

Nolen-Hoeksema, S., \& Morrow, J. (1991). A prospective study of depression and posttraumatic stress symptoms after a natural disaster: The 1989 Loma Prieta earthquake. Journal of Personality and Social Psychology, 61, 115-121.

Nolen-Hoeksema, S., \& Morrow, J. (1993). Effects of rumination and distraction on naturally occurring depressed mood. Cognition and Emotion, 7, 561-570.

Nolen-Hoeksema, S., Wisco, B., \& Lyubomirsky, S. (2008). Rethinking rumination. Perspectives on Psychological Science, 3, 400-424. 
Philippot, P., \& Brutoux, F. (2008). Induced rumination dampens executive processes in dysphoric young adults. Journal of Behavior Therapy and Experimental Psychiatry, 39, 219-227.

Treynor, W., Gonzalez, R., \& Nolen-Hoeksema, S. (2003). Rumination reconsidered: A psychometric analysis. Cognitive Therapy and Research, 27, 247-259.

Van Overshelde, J. P., Rawson, K. A., \& Dunlosky, J. (2004). Category norms: An updated and expanded version of the Battig and Montague (1969) norms. Journal of Memory and Language, 50, 289-335.
Watkins, E. (2008). Constructive and unconstructive repetitive thought. Psychological Bulletin, 134, 163-206.

Watkins, E., Baeyens, C. B., \& Read, R. (2009). Concreteness training reduces dysphoria: Proof-ofprinciple for repeated cognitive bias modification in depression. Journal of Abnormal Psychology, 118, $55-64$.

Watkins, E., \& Brown, R. (2002). Rumination and executive function in depression: An experimental study. Journal of Neurology, Neurosurgery, and Psychiatry, 72, 400-402. 